\title{
Chronic ischemic cerebral white matter disease is a risk factor for nonfocal neurologic injury after total aortic arch replacement
}

Ridwan Lin, MD, PhD, ${ }^{a}$ Lars Svensson, MD, PhD, ${ }^{\mathrm{b}}$ Rishi Gupta, $\mathrm{MD}^{\mathrm{c}}$ Bruce Lytle, MD, ${ }^{\mathrm{b}}$ and Derk Krieger, MD, $\mathrm{PhD}^{\mathrm{a}}$

From the Departments of Neurology and Thoracic and Cardiovascular Surgery, Center for Aortic Surgery, Marfan Syndrome and Connective Tissue Disorder Clinic, Cleveland Clinic, Cleveland, Ohio; and Department of Neurology, ${ }^{\mathrm{c}}$ Division of Cerebrovascular Diseases, Michigan State University, East Lansing, Mich.

Received for publication Sept 12, 2006; revisions received Nov 14, 2006; accepted for publication Nov 20, 2006.

Address for reprints: Lars Svensson, MD, $\mathrm{PhD}$, Department of Thoracic and Cardiovascular Surgery, Cleveland Clinic, 9500 Euclid Avenue/Desk F24, Cleveland, OH 44195 (E-mail: svenss1@ccf.org).

J Thorac Cardiovasc Surg 2007;133:1059-65 0022-5223/ $\$ 32.00$

Copyright $(9) 2007$ by The American Association for Thoracic Surgery

doi:10.1016/j.jtcvs.2006.11.036
Objective: Leukoaraiosis (chronic ischemic white matter changes) on preoperative brain magnetic resonance imaging is common in patients having aortic arch surgery. This study sought to determine whether it is associated with adverse neurologic outcome in the postoperative period.

Methods: Data were collected from a retrospective chart review of 142 patients in whom total aortic arch replacement was planned at the Cleveland Clinic between April 2000 and December 2004. All patients had preoperative brain magnetic resonance imaging evaluation. Leukoaraiosis severity was rated semiquantitatively using the Schelten's scale. Postoperative neurologic injuries were investigated by clinical examination and appropriate neuroimaging. They were stratified as type 1 (focal ischemic stroke) and type 2 (nonfocal neurocognitive changes, generalized seizures) injuries.

Results: The following were independent predictors of type 1 neurologic injury: age (odds ratio 1.06 [1.01-1.13], $P=.02$ ) and moderate to severe aortic atheroma (odds ratio 4.4 [1.4-9.7], $P=.012$ ). Total white matter scores (odds ratio 1.16 [1.06-1.27], $P=.002$ ) and higher preoperative hemoglobin A1c levels (odds ratio 1.8 [1.003.50], $P=.05$ ) were significantly associated with type 2 neurologic injuries. Survival was $96 \%$, and $4.2 \%$ had persistent focal neurologic deficits at the time of hospital discharge.

Conclusions: Leukoaraiosis is a significant independent predictor of nonfocal postoperative neurologic morbidity following aortic arch replacement surgery. Preoperative evaluation with magnetic resonance imaging allows identification of a patient subgroup at risk and implementation of strategies aimed at improving neurologic outcome.

$\mathrm{P}$ ostoperative neurologic complication is a serious concern in patients undergoing aortic arch replacement surgery. ${ }^{1-4}$ Identification of risk factors that adversely affect neurologic outcome is an important step toward reducing neurologic morbidity and mortality. Although history of symptomatic stroke has been described as a determinant of postoperative stroke and early mortality after aortic arch surgery, ${ }^{4}$ the significance of chronic ischemic cerebral white matter (WM) disease has not been clearly defined in the perioperative setting. Leukoaraiosis is a descriptive term for neuroimaging abnormalities of the WM, appearing as patchy or confluent subcortical and periventricular hypodensity on computed tomography or hyperintensity on T2-weighted magnetic resonance imaging (MRI). It reflects pathologic and chronic ischemic demyelination of WM tracts and, as such, is distinct from small vessel stroke of lacunar infarction. These silent brain lesions are often seen on brain MRI scans of elderly patients with hypertension, diabetes, with or without prior history of stroke or dementia. ${ }^{5-9}$ Prior studies suggest that leukoaraiosis predicts future risk of stroke and disability in the general popula- 

Abbreviations and Acronyms
$\mathrm{ACP}=$ antegrade cerebral protection
$\mathrm{CPB}=$ cardiopulmonary bypass
$\mathrm{CPBT}=$ cardiopulmonary bypass pump time
FLAIR = fluid-attenuated inversion recovery
MAP $=$ mean arterial pressure
MRI = magnetic resonance imaging
$\mathrm{OR}=$ odds ratio
$\mathrm{RCP}=$ retrograde cerebral protection
$\mathrm{WM}=$ white matter

tion. ${ }^{5,10-12}$ This study sought to determine whether these silent brain lesions present increased risks for adverse neurologic outcome in the postoperative period after aortic arch replacement surgery.

\section{Materials and Methods}

Demographic, clinical, and intraoperative data were collected from a retrospective chart review of 142 patients (143 cases) who had repair of the ascending and aortic arch between April 2000 and December 2004. Preoperative MRI evaluations were obtained on patients in whom the surgeons anticipated total arch replacement surgeries. All consecutive patients with preoperative MRI were analyzed. The study was approved by the Institutional Review Board of the Cleveland Clinic Foundation.

All MRI scans were acquired at the Cleveland Clinic using standard MRI protocol comprising axial T1-weighted, T2weighted, and fluid-attenuated inversion recovery (FLAIR) sequences (slice thickness $5 \mathrm{~mm}$, interslice gap $0.5 \mathrm{~mm}$ ). Subcortical WM lesions were visually rated using the semiquantitative Schelten's scale. ${ }^{13}$ This scale was previously validated for inter- and intraobserver agreement ${ }^{13}$ and showed moderate to good interobserver agreement compared with the Fazekas and Rotterdam Scan Study Scales on baseline cross-sectional assessments of WM lesions. ${ }^{14}$ Based on the Schelten method, WM hyperintensity was graded on brain T2-weighted or FLAIR MRI sequences based on the extent and confluency of WM signal abnormality in the periventricular and subcortical locations. Periventricular WM hyperintensity adjacent to the anterior, body, and posterior horns of the lateral ventricles was each graded 0 to 2 based on the thickness of "caps" or "bands" adjacent to the lateral ventricles. Subcortical WM hyperintensity in each of the frontal, parietal, temporal, and occipital lobes was graded 0 to 6 based on the number and confluency of the WM lesions. ${ }^{13}$ The subcortical and periventricular scores were added, yielding a total possible score of 30 . To differentiate from lacunar infarction, leukoaraiosis was defined on brain MRI as hyperintense T2 lesions without corresponding hypointense T1 lesions. Imaging analysis was performed by researchers blinded to the clinical and surgical details of the patients. Figure 1 shows the MRI scans of varying leukoaraiosis severity and their corresponding WM scores.

For the total arch replacements, patients were placed on cardiopulmonary bypass (CPB) with a side graft attached to the subclavian or axillary artery. Patients were cooled to a systemic temperature below $20^{\circ} \mathrm{C}$, and then antegrade or retrograde brain perfusion was established as indicated and reported previously. ${ }^{15}$ Our protocols for managing $\mathrm{CPB}$ and circulatory arrest have been described previously. ${ }^{16}$ Postoperative neurologic complications were determined by clinical examination by consulting neurologists and using appropriate neuroimaging. These were stratified into type 1 (focal ischemic strokes) and type 2 neurologic injuries (nonfocal encephalopathy and generalized seizures).

\section{Statistical Analysis}

Statistical analysis was conducted using the SPSS statistical package. Univariate associations between potential predictors were analyzed using a Fisher exact test for categorical variables and a Student $t$ test for continuous variables. Stepwise binary logistic regression was performed on variables with a value of $P \leq .20$ from the univariate analyses to determine independent predictors of neurologic injury postoperatively. Results were reported as odd ratios (ORs) with associated $95 \%$ confidence intervals.

\section{Results}

Demographic and clinical baseline characteristics of patients in the study are shown in Table 1. A total of 26 (18.2\%) central nervous system neurologic adverse events in the postoperative period were recorded, of which $4.2 \%$ had persistent focal neurologic deficits at the time of hospital discharge. The adverse neurologic events consisted of 15 type $1(10.5 \%)$ neurologic injuries; 9 (6.3\%) of these had improved or resolved to baseline at the time of hospital discharge. Eleven patients had type $2(7.7 \%)$ neurologic injuries; 6 of these $(4.2 \%)$ resolved at the time of hospital discharge. Patients with Marfan syndrome who underwent aortic arch replacement made up a subgroup $(n=9,6.3 \%)$ whose members on average were younger (43.4 \pm 13.3 years). Six of the Marfan patients $(67 \%)$ had hypertension, $5(55.6 \%)$ had hyperlipidemia, and $3(33.3 \%)$ had coronary artery disease (nonobstructive or single vessel disease). Leukoaraiosis was found in 4 patients $(44.4 \%)$. None of the Marfan patients had adverse neurologic events in the postoperative period.

Table 2 shows the independent predictors of adverse neurologic events in the postoperative period. Moderate to severe arch atheroma, hemoglobin A1c, and total WM score were associated with neurologic injury on multivariate analysis. When stratified based on the type of injury, age and moderate to severe aortic arch atheroma were significant independent predictors of type 1 neurologic injury, whereas higher WM score and preoperative hemoglobin A1c were associated with type 2 neurologic injury. Compared with type 1 neurologic injury, patients with type 2 injury were significantly more likely to have higher WM scores $(18 \pm 8$ vs $11 \pm 6, P<.007)$. Compared with type 2 injury, patients with type 1 injury were significantly older $(70 \pm 10$ vs $62 \pm$ 14 years, $P<.014$ ) and were more likely to have moderate 

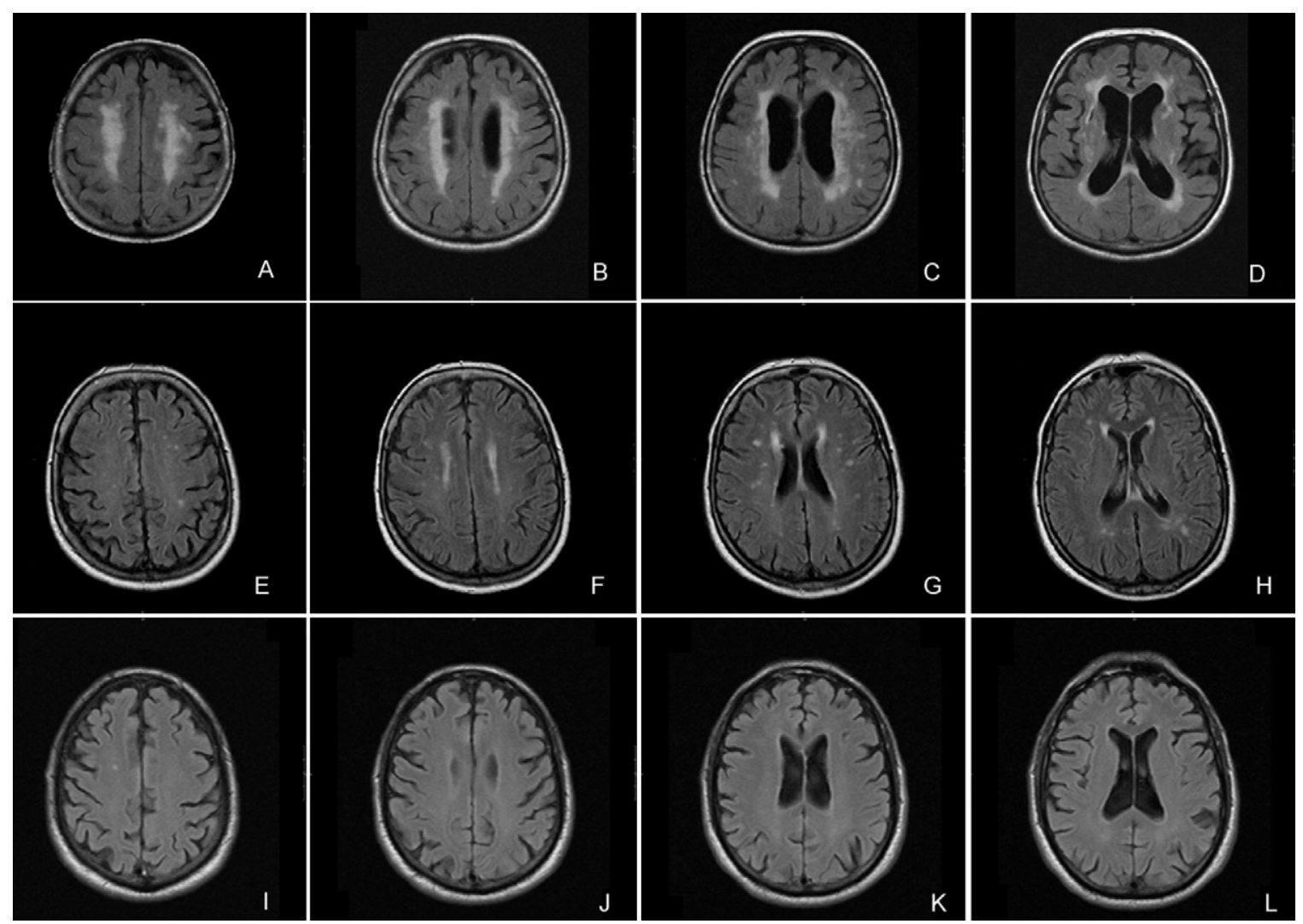

Figure 1. Composite MRI scans of 3 patients with varying degree of leukoaraiosis and corresponding total WM score based on the Schelten's rating method. A-D, Severe leukoaraiosis (WM score of 26). E-H, Moderate leukoaraiosis (WM score of 17). I-L, Minimal-mild leukoaraiosis (WM score of 3).

to severe atheroma on the aortic arch $(\mathrm{OR}=4.4$ [1.4-9.7], $P=.012)$. Hospital survival was $96 \%$ (136/142).

Patients who sustained neurologic injury were significantly more likely to have a higher length of hospitalization in comparison with those without neurologic injury (19.5 \pm 20.3 vs $9.5 \pm 5.1$ days, $P<.0001)$. Only $42 \%$ of patients with neurologic injury were discharged directly home rather than to a rehabilitation facility, although $91 \%$ of patients without neurologic injury were discharged home $(P=$ .0001). Table 3 shows that patients with a higher total WM score, moderate to severe arch atheroma, and a mean arterial pressure $(\mathrm{MAP})<50 \mathrm{~mm} \mathrm{Hg}$ during surgery were less likely to be discharged directly to home.

On multivariate analysis, none of the intraoperative variables analyzed was independently associated with neurologic injury (data not shown). Analysis of cerebral protection with respect to neurologic outcome in these patients showed no difference between antegrade cerebral protection (ACP; 10.53\%) and retrograde cerebral protection (RCP; $16.67 \%, P=.31)$. However, all 4 neurologic events with the ACP method were comprised of type 1 injury, whereas only 3 of 5 neurologic events with the RCP method were focal type 1 injury.

\section{Discussion}

The field of aortic arch surgery has evolved with the introduction of new techniques and technologies. ${ }^{1-3,15-18}$ Prevention of neurologic deficits remains an important goal for successful total aortic arch repair. The important finding in this study is that patients with severe leukoaraiosis were more likely to develop postoperative nonfocal neurologic events along with a lower probability of being discharged directly home. The risks were incrementally based on the severity of WM score, conferring up to a 4.8-fold risk at the extreme ends of WM severity.

Leukoaraiosis is a common neuroimaging finding in elderly and hypertensive patients with small-vessel ischemic cerebrovascular disease. Although population studies (Cardiovascular Health Study ${ }^{11}$ and Rotterdam Scan Study ${ }^{10}$ ) suggest that leukoaraiosis independently predicts increased stroke risk in the general population, its impact on postoperative neurologic outcome after cardiovascular surgery has 
TABLE 1. Baseline patient characteristics

\begin{tabular}{|c|c|}
\hline$\overline{A g e}(y \pm S D)$ & $63( \pm 14)$ \\
\hline Male, n (\%) & $89(61.8 \%)$ \\
\hline Hypertension, n (\%) & $122(84.7 \%)$ \\
\hline Hyperlipidemia, n (\%) & $97(67.4 \%)$ \\
\hline Diabetes mellitus, $\mathrm{n}(\%)$ & $14(9.7 \%)$ \\
\hline Average preoperative hemoglobin $\mathrm{A} 1 \mathrm{c}( \pm \mathrm{SD})$ & $5.40( \pm 0.9)$ \\
\hline Coronary artery disease, $\mathrm{n}(\%)$ & $88(61.1 \%)$ \\
\hline $\begin{array}{l}\text { Preoperative left ventricle ejection fraction, } \\
\quad \%( \pm S D)\end{array}$ & $54.4 \%( \pm 7 \%)$ \\
\hline Atrial fibrillation, $\mathrm{n}(\%)$ & $23(16.0 \%)$ \\
\hline Valvular disease, $\mathrm{n}(\%)$ & $114(79.2 \%)$ \\
\hline History of transient ischemic attack, $\mathrm{n}(\%)$ & $21(14.6 \%)$ \\
\hline History of symptomatic stroke, $\mathrm{n}(\%)$ & $14(9.7 \%)$ \\
\hline Presence of leukoaraiosis, n (\%) & $105(72.9 \%)$ \\
\hline Peripheral vascular disease, $\mathrm{n}(\%)$ & $18(12.5 \%)$ \\
\hline \multicolumn{2}{|l|}{ Tobacco use, n (\%) } \\
\hline Never/previous use & $128(90.8 \%)$ \\
\hline Current & $13(9.2 \%)$ \\
\hline Pulmonary disease, $\mathrm{n}(\%)$ & $35(24.3 \%)$ \\
\hline Renal failure, $\mathrm{n}(\%)$ & $8(5.6 \%)$ \\
\hline \multicolumn{2}{|l|}{ Aortic arch disease type, $\mathrm{n}(\%)$} \\
\hline Aneurysm & $80(55.6 \%)$ \\
\hline Dissection & $1(0.7 \%)$ \\
\hline Dissection + aneurysm & $63(43.8 \%)$ \\
\hline Coarctation + aneurysm & $2(1.4 \%)$ \\
\hline \multicolumn{2}{|l|}{ Type of aortic dissection, $\mathrm{n}(\%)$} \\
\hline Stanford type A & $36(25 \%)$ \\
\hline Stanford type B & $28(19.4 \%)$ \\
\hline \multicolumn{2}{|l|}{ Severity of aortic atheroma, $\mathrm{n}(\%)$} \\
\hline None-mild & $93(65.0 \%)$ \\
\hline Moderate-severe & $50(35.0 \%)$ \\
\hline \multicolumn{2}{|l|}{ Surgery timing, $\mathrm{n}(\%)$} \\
\hline Elective & $141(97.9 \%)$ \\
\hline Emergent & $3(2.08 \%)$ \\
\hline \multicolumn{2}{|l|}{ Type(s) of surgery, n (\%) } \\
\hline Ascending or total arch & $43(29.9 \%)$ \\
\hline Arch + valve replacement surgery & $42(29.2 \%)$ \\
\hline Arch + CABG & $12(8.3 \%)$ \\
\hline Arch + valve surgery + CABG & $21(14.6 \%)$ \\
\hline Others (arch + aortic endarterectomy, Maze) & $26(18.1 \%)$ \\
\hline
\end{tabular}

$C A B G$, coronary artery bypass grafting; $S D$, standard deviation.

not been clearly studied. With total aortic arch replacements, we found that leukoaraiosis was associated with global type 2 but not focal type 1 ischemic events. Focal type 1 event was more prevalent in patients with moderate to severe aortic arch atheroma, consistent with previous studies demonstrating that aortic atherosclerosis is a risk factor for focal ischemic stroke after cardiac surgery. ${ }^{19} \mathrm{On}$ the other hand, encephalopathy and seizures associated with global type 2 injuries were more prevalent in patients with severe and diffuse leukoaraiosis. The lack of association between neurocognitive changes and aortic atherosclerosis has been previously reported by the Neurologic Outcome
TABLE 2. Independent predictors of neurologic injury after aortic arch surgery

\begin{tabular}{llll}
\hline Variable & OR & 95\% Cl & P value \\
\hline Any neurologic injury & & & \\
$\quad$ Moderate-severe arch atheroma & 2.5 & $1.2-6.2$ & .017 \\
$\quad$ Hemoglobin A1c & 2.3 & $1.2-4.2$ & .010 \\
$\quad$ Total white matter score & 4.1 & $1.1-15.1$ & .030 \\
Type 1 neurologic injury & & & \\
$\quad$ Age & 1.06 & $1.01-1.13$ & .02 \\
$\quad$ Moderate-severe arch atheroma & 4.4 & $1.4-9.7$ & .012 \\
$\begin{array}{l}\text { Type 2 neurologic injury } \\
\quad \text { Total white matter score }\end{array}$ & 1.16 & $1.06-1.27$ & .002 \\
$\quad$ Hemoglobin A1c & 1.8 & $1.00-3.5$ & .05 \\
\hline
\end{tabular}

$O R$, odds ratio; $C l$, confidence interval.

Research Group and CARE investigators. ${ }^{20}$ It suggests that other mechanisms, such as hypoperfusion or microembolic events, may be significant factors underlying this type of injury. The latter is generally attributed to gaseous bubbles and/or other smaller particulate matter that are associated with the CPB procedure. ${ }^{21}$ Patients with severe leukoaraiosis may be more susceptible to the effects of anesthesia, metabolic derangement, sedating medications, hypoxia, or subtle cerebral hemodynamic shifts that were not detectable with our conventional MRI protocol.

Why patients with advanced leukoaraiosis have higher likelihood of global type 2 injuries after aortic surgery is not clear. Postmortem and imaging studies showed that the density of small penetrating vessels ${ }^{22}$ and regional cerebral blood flow in the ischemic $\mathrm{WM}^{23,24}$ were reduced in patients with leukoaraiosis. These patients may have a lower symptomatic threshold to the effects of microemboli or hemodynamic shifts during CPB. Impaired subcortical WM vascular reserve may also make patients with severe leukoaraiosis more susceptible to the metabolic effects of hyperglycemia and the global effects of sedating medications. Patients with diabetes have been shown to have abnormal cerebral autoregulation during CPB. ${ }^{25}$

Previous studies of neurologic deficits in patients having percutaneous interventions, vascular surgery, or cardiac surgery have not examined preoperative MRI evidence of leukoaraiosis, and this may be the reason for the poor

TABLE 3. Independent predictors of patient not discharged directly to home

\begin{tabular}{llrc}
\hline & OR & 95\% Cl & $\boldsymbol{P}$ value \\
\hline Total white matter score & 1.19 & $1.08-1.32$ & .001 \\
Moderate-severe arch atheroma & 3.35 & $1.01-11.2$ & .049 \\
Intraoperative MAP $<50 \mathrm{~mm} \mathrm{Hg}$ & 6.1 & $1.4-27.7$ & .019
\end{tabular}

$\mathrm{Cl}$, confidence interval; $M A P$, mean arterial pressure; $O R$, odds ratio. 
correlation between procedures and postprocedural neurocognitive deficits. This raises the issue that any patient with cardiovascular disease is likely to have chronic cerebral WM disease, and treatment of any index disease (for example, coronary or carotid disease) may unmask a previously silent or compensated cerebral ischemic process. This study suggests that preoperative MRI finding of leukoaraiosis may be a risk factor of any cardiac surgery and likely of other interventional/surgical procedures. Indeed, in our previous prospective study, we found $38 \%$ of patients having aortic arch operations had unexplained preoperative neurocognitive deficits, ${ }^{15}$ including the control patients having coronary artery bypass in the study.

In this study, intraoperative variables did not independently predict neurologic injury after aortic arch repair (data not shown). In contrast, in a previous investigation, $\mathrm{CPB}$ pump time (CPBT) and circulatory arrest time were found to be predictive for perioperative stroke in univariate analysis, and CPBT was an independent predictor of perioperative stroke in the multivariate analysis. ${ }^{4}$ Recent introduction of multimodal cerebral protection protocol $^{16}$ and shorter average CPBT in the current study (mean 108 minutes, SD 41 vs 163 minutes, SD 52, in the previous study) may account for the different findings. A smaller sample size in the current study may also limit our ability to detect the complex interplay between various intraoperative factors.

Neurologic injury after aortic arch replacement surgery has a significant impact on prognosis. In our study, 4 postoperative mortalities $(2.8 \%)$ were associated with neurologic complications. Neurologic injury was associated with prolonged hospitalization, lower likelihood of being discharged directly to home, and increased need for rehabilitation. Using inability to discharge to home after surgery and the need for rehabilitation as the surrogate index of disability, we found that both leukoaraiosis and moderate to severe aortic atheroma independently predicted worse outcome. Intraoperative MAP $<50 \mathrm{~mm} \mathrm{Hg}$ was also an independent predictor of inability to be discharged home, although it did not predict neurologic injury. It is possible that intraoperative hypoperfusion affecting other organ systems, such as renal hypoperfusion and renal failure, may account for this association.

Progression of leukoaraiosis has been associated with substantial morbidity due to mobility decline ${ }^{26-28}$ and falls, ${ }^{29}$ cognitive dysfunction, ${ }^{30}$ stroke, ${ }^{10,11}$ and vascular deaths. ${ }^{31}$ Whether the use of CPB alters progression of leukoaraiosis is an interesting question that is a subject for future study. The pathophysiology of small vessel disease suggests that subcortical ischemia may be unmasked during the low-flow state of CPB. Patients with severe WM disease burden likely have lower tolerance to intraoperative hypoperfusion, microemboli, and postoperative hemodynamic, metabolic, and medication effects. Close attention to these variables and future prospective and systematic studies are needed to design preventive approaches to reduce postoperative morbidity. For example, maintaining higher perfusion pressure during $\mathrm{CPB}$ in patients with severe leukoaraiosis might be protective. Although we did not find average intraoperative MAP to be an independent predictor of neurologic injury in our study, measurement of average MAP might not be a sensitive indicator of brain perfusion pressure. The present study suggests that leukoaraiosis is a useful marker for identifying patients at risk for global neurologic injury during aortic arch replacement surgery.

There are several limitations inherent in the current study. Our study lacked formal neurocognitive assessment, which is a limitation inherent in the retrospective nature of this study. All of the neurologic events noted as type 1 and type 2 events have been formally assessed by consulting neurologists and verified with imaging as necessary. In all patients, the postoperative encephalopathy represented an overt change from their preoperative baseline. The incidence rate was $7.7 \%$, which was significantly lower than the reported incidence of neurocognitive changes after cardiac surgery in the literature. Subtle cognitive impairment that was not ascertained in this study could have underestimated the extent of neurocognitive injury. Another limitation is the focus on a patient population having complex procedures such as total arch replacement, with or without concurrent coronary bypass or valve surgeries. Although we cannot rule out the possibility of bias, $24.3 \%$ of patients in the study had histories of transient ischemic attack or clinical stroke, which we believe make systematic bias due to overrepresentation of high-risk neurologic patients seem less likely. Third, the limited focus on neurocognitive dysfunction in the early postoperative period without longer-term assessment is another limitation of this study. A previous study has shown that patients with perioperative neurocognitive injury were more likely to have long-term neurocognitive disability and decreased overall quality of life. ${ }^{32}$ Nevertheless, in our previous prospective study of neurocognitive function after aortic arch replacement, we found that by 6 months, all the patients with new deficits (9\%) had recovered and had done no worse on mean scores than control patients having coronary artery bypass surgery. ${ }^{15}$ Differences in cognitive measures used may explain these conflicting findings. The long-term effect of postoperative neurocognitive dysfunction in patients with severe leukoaraiosis will need to be assessed in a prospective manner using standardized cognitive measures that account for practice effect. ${ }^{15}$

Despite these limitations, we feel that the impact of leukoaraiosis on postoperative neurocognitive functioning is a clinically important factor for risk assessment during preoperative evaluation. It needs to be tested in a larger prospective study with formal neurocognitive testing to determine the total WM score thresholds of concern. Quantitative microembolic event monitoring during surgery may be helpful to determine 
whether there are varying thresholds for postoperative neurocognitive dysfunction in patients with different WM scores on preoperative MRI. This study suggests that MRI evidence of leukoaraiosis may be used as a marker for chronic ischemic cerebrovascular disease in identifying patients at risk for global neurologic injury during aortic arch surgery.

\section{References}

1. Svensson LG. Progress in ascending and aortic arch surgery: minimally invasive surgery, blood conservation, and neurological deficit prevention. Ann Thorac Surg. 2002;74:S1786-8.

2. Okita Y, Minatoya K, Tagusari O, Ando M, Nagatsuka K, Kitamura S Prospective comparative study of brain protection in total aortic arch replacement: deep hypothermic circulatory arrest with retrograde cerebral perfusion or selective antegrade cerebral perfusion. Ann Thorac Surg. 2001;72:72-9.

3. Hagl C, Ergin MA, Galla JD, Landsman SL, McCullough JN, Speilvogel D, et al. Neurologic outcome after ascending aorta-aortic arch operations: effect of brain protection technique in high-risk patients. J Thorac Cardiovasc Surg. 2001;121:1107-21.

4. Svensson LG, Crawford ES, Hess KR, Coselli JS, Raskin S, Shenaq SA, et al. Deep hypothermia with circulatory arrest: determinants of stroke and early mortality in 656 patients. J Thorac Cardiovasc Surg. 1993;106:19-30.

5. Leys D, Englund E, Del Ser T, Inzitari D, Fazekas F, Bornstein N, et al,on behalf of the European Task Force on Age-related White Matter Changes. White matter changes in stroke patients. Eur Neurol. 1999; 42:67-75.

6. Van der Flier WM, van Straaten ECW, Barkhof F, Verdelho A, Madureira S, Pantoni L, et al, on behalf of the LADIS Study Group. Small vessel disease and general cognitive function in nondisabled elderly. Stroke. 2005;36:2116-20.

7. De Leeuw FE, De Groot JC, Achten E, Oudkerk M, Ramos LMP, Jeijboer R, et al. Prevalence of cerebral white matter lesions in elderly people: a population based magnetic resonance imaging study. The Rotterdam Scan Study. J Neurol Neurosurg Psychiatry. 2001;70:9-14.

8. Longstreth WT Jr, Diehr P, Manolio TA, Beauchamp NJ, Jungreis CA, Lefkowitz D, for the Cardiovascular Health Study Collaborative Research Group. Cluster analysis and patters of findings on cranial magnetic resonance imaging of the elderly. Arch Neurol. 2001;58:635-40.

9. Jeerakathil T, Wolf PA, Beiser A, Massaro J, Seshadri S, D’Agostino $\mathrm{RB}$, et al. Stroke risk profile predicts white matter hyperintensity volume. The Framingham Study. Stroke. 2004;35:1857-61.

10. Vermeer SE, Hollander M, Van Dijk EJ, Hofman A, Koudstaal PJ, Breteler MMB. Silent brain infarcts and white matter lesions increase stroke risk in the general population. Stroke. 2003;34:1913-6.

11. Kuller LH, Longstreth WT, Arnold AM, Bermick C, Bryan N, Beauchamp N, for the Cardiovascular Health Study Collaborative Research Group. White matter hyperintensity on cranial magnetic resonance imaging: a predictor of stroke. Stroke. 2004;35:1821-25.

12. Henon H, Vroylandt P, Durieu I, Pasquier F, Leys D. Leukoaraiosis more than dementia is a predictor of stroke recurrence. Stroke. 2003; 34:2935-40.

13. Scheltens P, Barkhof F, Leys D, Pruvo JP, Nauta JJP, Vermersch P, et al. A semiquantitative rating scale for the assessment of signal hyperintensities on magnetic resonance imaging. J Neurol Sci. 1993;114:712.

14. Prins ND, Van Straaten ECW, Van Dijk EJ, Simoni M, Van Schijndel RA, Vrooman HA, et al. Measuring progression of cerebral white matter lesions on MRI. Visual rating and volumetrics. Neurology. 2004;62:1533-9.

15. Svensson LG, Nadolny E, Penney DL, Jacobson J, Kimmel WA, Entrup MH, et al. Prospective randomized neurocognitive and S-100 study of hypothermic circulatory arrest, retrograde brain perfusion, and antegrade brain perfusion for aortic arch operations. Ann Thorac Surg. 2001;71:1905-12.
16. Svensson LG, Nadolny EM, Kimmel WA. Multimodal protocol influence on stroke and neurocognitive deficit prevention after ascending/ arch aortic operations. Ann Thorac Surg. 2002;74:2040-6.

17. Safi H, Miller C, Estrera A, Huynh T, Rubenstein FS, Subramaniam $\mathrm{MH}$, et al. Staged repair of extensive aortic aneurysms. Circulation. 2001;104:2938-42.

18. Shimazaki Y, Watanabe T, Takahashi T, Minowa T, Inui K, Uchida T, et al. Minimized mortality and neurological complications in surgery for chronic arch aneurysm: axillary artery cannulation, selective cerebral perfusion, and replacement of the ascending and total arch aorta. J Card Surg. 2004;19:338-42.

19. Mackensen GB, Ti LK, Phillips-Bute BG, Mathew JP, Newman MF, Grocott HP, and the Neurologic Outcome Research Group (NORG). Cerebral embolization during cardiac surgery: impact of aortic atheroma burden. Br J Anaesth. 2003;91:656-61.

20. Bar-Yosef S, Anders M, Mackensen GB, Ti LK, Mathew JP, PhillipsBute B, et al, for theNeurological Outcome Research Group and CARE Investigators of the Duke Heart Center. Aortic atheroma burden and cognitive dysfunction after coronary artery bypass graft surgery. Ann Thorac Surg. 2004;78:1556-62.

21. Borger MA, Feindel CM. Cerebral emboli during cardiopulmonary bypass: effect of perfusionist interventions and aortic cannulas. $J$ ExtraCorp Tech. 2002;34:29-33.

22. Moody DM, Thore CR, Anstrom JA, Challa VR, Langefeld CD, Brown WR. Quantification of afferent vessels shows reduced brain vascular density in subjects with leukoaraiosis. Radiology. 2004;233:883-90.

23. Oishi M, Mochizuki Y. Regional cerebral blood flow and cerebrospinal fluid glutamate in leukoaraiosis. J Neurol. 1998;245:777-80.

24. Markus HS, Lythgoe DJ, Ostegaard L, O'Sullivan M, Williams SCR. Reduced cerebral blood flow in white matter in ischaemic leukoaraiosis demonstrated using quantitative exogenous contrast based perfusion MRI. J Neurol Neurosurg Psychiatry. 2000;69:48-53.

25. Croughwell N, Lyth M, Quill T, Newman M, Greeley W, Smith R, et al. Diabetic patients have abnormal cerebral autoregulation during cardiopulmonary bypass. Circulation. 1990;82(suppl IV):IV407-12.

26. Benson RR, Gutmann CRG, Wei X. Older people with impaired mobility have specific loci of periventricular abnormality on MRI. Neurology. 2002;58:48-55.

27. Onen F, Feugeas MCH, Baron G, De Marco G, Godon-Hardy S, Peretti II, et al. Leukoaraiosis and mobility decline: a high resolution magnetic resonance imaging study in older people with mild cognitive impairment. Neurosci Lett. 2004;355:185-8.

28. Briley DP, Wasay M, Sergent S, Thomas S. Cerebral white matter changes (leukoaraiosis), stroke and gait disturbance. J Am Geriatr Soc. 1997;45:1434-8.

29. Briley DP, Haroon S, Sergent S, Thomas S. Does leukoaraiosis predict morbidity and mortality? Neurology. 2000;54:90-4.

30. Wen HM, Mok VCT, Fan YH, Lam WWM, Tang WK, Wong A, et al. Effect of white matter changes on cognitive impairment in patients with lacunar infarcts. Stroke. 2004;35:1826-30.

31. Inzitari D, Cadelo M, Marranci ML, Pracucci G, Pantoni L. Vascular deaths in elderly neurological patients with leukoaraiosis. J Neurol Neurosurg Psychiatry. 1997;62:177-81.

32. Newman MF, Grocott HP, Mathew JP, White WD, Landolfo K, Reves JG, et al, for the Neurologic Outcome Research Group and the Cardiothoracic Anesthesia Research Endeavors (CARE) Investigators of the Duke Heart Center. Report of the substudy assessing the impact of neurocognitive function on quality of life 5 years after cardiac surgery. Stroke. 2001;32:2874-81.

\section{Appendix}

A list of preoperative and intraoperative variables analyzed follows.

\section{Preoperative Variables}

Age

Gender

Hypertension

Hyperlipidemia 
Diabetes mellitus; average preoperative hemoglobin A1c

Coronary artery disease

Left ventricle ejection fraction

A trial fibrillation

Valvular disease

Prior transient ischemic attack

Prior stroke

Presence of leukoaraiosis on brain MRI

White matter score

Peripheral vascular disease

Tobacco use

Never/previous use

Current

Pulmonary disease

Renal failure

Aortic arch disease type

Aneurysm

Dissection

Dissection + aneurysm

Coarctation + aneurysm

Type of aortic dissection

Stanford type A

Stanford type B
Severity of aortic atheroma

None to mild

Moderate to severe

Surgery timing

Elective

Emergen

Type(s) of surgery

Ascending or total arch

Arch + valve replacement surgery

Arch + coronary artery bypass grafting

Arch + valve surgery + coronary artery bypass grafting

Others (arch + aortic endarterectomy, Maze)

Intraoperative Variables

Total CPB time

Total arrest time

Total crossclamp time

Degree of hypothermia

Rewarming time

Use of antegrade/retrograde cerebral protection

Antegrade

Retrograde

Average intraoperative MAP

Preoperative hematocrit

Postoperative hematocrit 